\title{
The birds of Sehlabathebe National Park, Lesotho
}

\section{G. KoPIJ}

Kopij, G. 2002. The birds of Sehlabathebe National Park, Lesotho. Koedoe 45(1): 65-78. Pretoria. ISSN 0075-6458.

A total of 117 bird species has been recorded in Sehlabathebe National Park, south-east Lesotho, consisting of 29 vagrants, 18 visitors and 70 breeding and probable breeding residents. For each species status was determined and abundance roughly estimated. Quantitative studies on breeding bird communities were carried out by means of the line transect method on four transects with the total length ca $30 \mathrm{~km}$ in the park and on two transects with the total length of ca $20 \mathrm{~km}$ outside the park. In the park, dominant species were represented by the Stonechat Saxicola torquata, Ayres' Cisticola Cisticola ayresii, Yellow-rumped Widow Euplectes capensis and Wailing Cisticola Cisticola lais. Outside the park dominants were represented by Cape Weaver Ploceus capensis, Cape Sparrow Passer melanurus, Cape Canary Serinus canicollis, Common Quail Coturnix coturnix, Stonechat, Cape Butning Emberiza capensis and Drakensberg Siskin Pseudochloroptila symonsi. Characteristic, high-altitude species in the park included Drakensberg Siskin, Mountain Pipit Anthus hoeschi, Orange-breasted Rockjumper Chaetops auriantius, Banded Martin Riparia cincta and Sentinel Rock Thrush Monticola explorator. Species such as the Laughing Dove Streptopelia senegalensis, Sicklewinged Chat Cercomela sinuata, Mountain Chat Oenanthe monticola, Thick-billed Lark Galerida magnirostris, Red-winged Starling Onychognathus morio, Alpine Swift Apus melba, Cape Sparrow, Grey-headed Sparrow Passer diffusus, Red Bishop Euplectes orix and Golden Bishop Euplectes afer were absent or occurred in very low densities in the park, although they are widespread and common in the Maluti/Drakensberg grasslands (including areas neighbouring to the park). The lack of trees and shrubs for nesting, the lack of cultivated fields as feeding places and competition with related species both for food and nesting sites, may partly play a role in this regard.

Key words: Sehlabathebe National Park, Lesotho, birds, quantitative studies, conservation.

G. Kopij, Department of Biology, National University of Lesotho, P. O. Roma 180, Lesotho.

\section{Introduction}

Sehlabathebe National Park (SNP) was proclaimed on 27 February 1970 and to date it is the only national park in Lesotho (Pomela et al. 2000). Plant diversity of SNP has been studied by Kopij (2001b), and animal diversity by Lynch \& Watson (1990) and Kopij (2001b). The first ornithological observations in SNP were made during the years 1975-1982 by J. Belcomb, C. Brown, R. Howard, M. Howard and L. Passineau. From 1987 to 1990 , a total of 16 visits to record birds of SNP were conducted. Each visit lasted 1-4 days (11 visits in summer (November-February) and five in winter (April-September)). The following persons participated in this project: L. P. Abbott, E. Bolding, J. Cambridge, K. E. Deas, S. Fairman, J. M. Gosnell, R. Guy, S. Haines, J. Jones, P. Osborne, D. P. Smits, B. Tigar, S. Van der Veur, J. P. Watson, H. Wilson, A. Wolfromm and $\mathrm{H}$. Wrayner. These observers used the standard Southern African Bird Atlas Project cards for reporting their records and also provided details of unusual records. To date, results of these observations are available mainly in the form of unpublished reports (Howard 1977; Howard \& Howard 1977; Belcomb 1980). Although a booklet on birds of the park was compiled by Passineau (1977), this is only a popular account of more common species, together with a simple, not 
annotated and not critically evaluated checklist of all species. The present study aims to determine and report on the status (based on my own and all previous records) and abundance (based on my own studies) of bird species in SNP and the surrounding area. To date, quantitative studies of bird communities in Lesotho have only been conducted in the lowlands: in urbanised areas of Maseru (Kopij 2000) and in rural areas and in farmlands in the Roma Valley (Kopij 2001a).

\section{Study area}

Located in the Qacha's Nek district of southeastern Lesotho, SNP (7239 ha) was established in 1970. Ranging in elevation from $2200 \mathrm{~m}$ to $2600 \mathrm{~m}$, it falls entirely within the Themeda-Festuca alpine grassland. SNP comprises short grasses (69\%), escarpments and colluvial lands (13\%), wetlands (11\%), water-courses $(4 \%)$ and roads and other man-modified areas $(3 \%)$. Except for a few small isolated willow Salix subserrata clumps, no other woody vegetation occurs in the park. Water in the form of streams, rivers and pools is abundant. Mean annual rainfall is $769 \mathrm{~mm}$, with most rains $(75 \%)$ in November-March. Mean annual maximum and minimum temperatures are $19.6^{\circ} \mathrm{C}$ and $2.2{ }^{\circ} \mathrm{C}$ respectively (Kopij 2001b). A map of SNP was published by Lynch \& Watson (1990).

\section{Methods}

\section{Quantitative studies}

Quantitative studies were carried out from 12 to 15 January 2001. The American version of the line transect method (Bibby et al. 1992) was employed to assess density and dominance of breeding species. The observer walked slowly, counting his paces and stopping often to observe and record birds. Only the birds in front and on either side of the observer were recorded - those behind were not. Where the transect ended, birds beyond the end point were not counted. Distant birds only detectable using binocular were not searched for. A pair, not an individual, was the censused unit. A singing or otherwise observed male, in a habitat suitable for breeding, was interpreted as one pair. If a male was not observed, a female, a group of fledglings or an occupied nest was also interpreted as one pair (Koskimies \& Vaisanen 1991; Bibby et al. 1992).

Density estimates are based on morning counts only, as birds were markedly less active in the afternoon. For calculation of relative abundance (dominance), morning and afternoon counts were pooled. For comparison of the breeding bird communities in the park with that in man-modified neighbouring areas, two transects were also undertaken outside the park.

Morning counts covered four transects:

- along the road from the research station to the lodge $(9 \mathrm{~km})$; grassland with numerous stream valleys at the foot of Thaba Ntšo; surveyed from 6:00 to $11: 00$ on 12 January 2001;

- from the research station along the north-west border of the park through Koti-se-Seholo Tsoelikane to Tsoelikane Falls (7 km); mainly short grassland with a few stream valleys and sandstone formations; surveyed from 6:00 to 10:00 on 13 January 2001;

- along the Tsoelikane River from Letamong village to the waterfall $(6 \mathrm{~km})$; extensive marshland with fairly tall and dense grass and sedges; surveyed from 10:00 to 12:30 on 13 January 2001;

- from the research station through the north-western corner of the park, Herdboy and Central Valleys to the waterfall $(9 \mathrm{~km})$; grassland with sandstone formations and stream valleys; surveyed from 6:00 to 11:00on 15 January.

Afternoon counts were conducted along three transects:

- from the lodge to the research station; short grass with numerous stream valleys, ca $0.5 \mathrm{~km}$ to $2 \mathrm{~km}$ south of the main road; surveyed from 12:00 to $16: 00$ on 12 January 2001;

- from the waterfall along Romoluma and Moseha to the research station; short grass with numerous sandstone formations; surveyed from 13:00 to $16: 00$ on 13 January 2001 ;

- from the waterfall to the lodge along Tsoelikane valley (marshland dominated by Merxmuelleria spp. with seven rock pools vegetated with Typha capensis, Aponogeton ranunculiflorus and other water plants); from the lodge to the research station through short grass with stream valleys south of the main road; surveyed from 12:00 to 16:00 on 15 January 2001.

Outside the park counts were conducted on two transects:

- $\quad$ along the road between the main gate to the park and the crossroad near the Roman Catholic church in Sehlabathebe (ca $10 \mathrm{~km}$ ); pastures, two villages, arable fields of wheat, sorghum and peas; surveyed from 9:45 to $12: 15$ on 
14 January 2001 ;

- along the Leqooa valley upstream from the bridge between Ha Mavuka and Ha Semenyane to the confluence of Leqooa River and Thamathu River near Ha Soloja; Salix trees grow along the river banks; the valley is partly cultivated (mainly with wheat) and marshland is utilised as pasture for cattle and horses; surveyed from 12:45 to $15: 45$ on 15 January 2001.

Dominant species are those represented by more than $5.0 \%$, and subdominant species by $2-4.9 \%$ of all breeding pairs recorded.

\section{Annotated checklist}

The checklist is based on results of all unpublished records by Howard (1977), Howard \& Howard (1977), Passineau (1977) and Balcomb (1980) and those of G. Kopij (this study)

The following criteria were used to determine the status and abundance of each species:

- Status: vagrant - single records or once in a five-year period; visitor - not resident, and nonbreeding, but recorded regularly in each year, often resident in neighbouring areas; resident direct breeding evidence (nest with eggs or chicks, egg shells); probable resident - indirect breeding evidence (occupied territory, simultaneously singing males in optimal habitats, breeding display etc.); Palearctic migrant breeds in the Palearctic region during the northern summer, wintering in SNP during the austral summer; intra-African migrant - breeds in SNP, but winters in tropical Africa; altitudinal migrant - breeding in SNP during the austral summer, but wintering outside the park, at lower altitudes; nomad - appears erratically, at no particular season, sometimes in large numbers that may breed.

- Abundance: very common-more than 200 breeding pairs (b.p.) in the entire park; common: 70-200 b.p.; fairy common - 20-69 b.p.; uncommon: $6-19$ b.p.; rare $-<5$ breeding pairs. Abundance has been roughly estimated through extrapolations from transect counts and from observations.

\section{Results of quantitative studies}

Among 1120 potential breeding pairs represented by 51 species recorded during 12 to 15 January 2001 in Sehlabathebe National Park, four species were classified as dominant: Stonechat, Ayres' Cisticola, Yellow-
Table 1

Relative abundance (dominance) of birds in Afroalpine Grassland in Sehlabathebe National Park in January 2001. Dominant species are given in bold case. Data from all transects is pooled

\begin{tabular}{|c|c|c|}
\hline Species & Pairs & $\begin{array}{c}\text { Dominance } \\
(\%)\end{array}$ \\
\hline Stonechat & 127 & 11.3 \\
\hline Ayres' Cisticola & 125 & 11.2 \\
\hline Yellow-rumped Widow & 95 & 8.5 \\
\hline Wailing Cisticola & 77 & 6.9 \\
\hline Drakensberg Siskin & 54 & 4.8 \\
\hline Mountain Pipit & 54 & 4.8 \\
\hline Long-tailed Widow & 50 & 4.5 \\
\hline Levaillant's Cisticola & 46 & 4.1 \\
\hline Orange-throated Longclaw & 44 & 3.9 \\
\hline Common Quail & 36 & 3.2 \\
\hline Black Swift & 34 & 3.0 \\
\hline Cape Bunting & 33 & 2.9 \\
\hline Sentinel Rock Thrush & 32 & 2.9 \\
\hline Cape Wagtail & 31 & 2.8 \\
\hline Cape Canary & 29 & 2.6 \\
\hline Orange-breasted Rockjumper & r 26 & 2.3 \\
\hline Ground Woodpecker & 24 & 2.1 \\
\hline Greater Striped Swallow & 24 & 2.1 \\
\hline Quail Finch & 22 & 2.0 \\
\hline Cape Weaver & 21 & 1.9 \\
\hline Banded Martin & 19 & 1.7 \\
\hline Malachite Sunbird & 13 & 1.2 \\
\hline Jackal Buzzard & 13 & 1.2 \\
\hline Cape Robin & 9 & 0.8 \\
\hline White-breasted Swallow & 8 & 0.7 \\
\hline Rock Pigeon & 6 & 0.5 \\
\hline Red-collared Widow & 5 & 0.4 \\
\hline Grey-wing Francolin & 5 & 0.4 \\
\hline Horus Swift & 5 & 0.4 \\
\hline Yellow-breasted Pipit & 5 & 0.4 \\
\hline Rock Kestrel & 5 & 0.4 \\
\hline Black Crow & 5 & 0.4 \\
\hline Rock Martin & 4 & 0.4 \\
\hline Black Duck & 3 & 0.3 \\
\hline Hadeda Ibis & 3 & 0.3 \\
\hline Yellow-billed Duck & 3 & 0.3 \\
\hline Red-winged Starling & 3 & 0.3 \\
\hline Cape Sparrow & 3 & 0.3 \\
\hline Pied Starling & 3 & 0.3 \\
\hline Common Waxbill & 3 & 0.3 \\
\hline Cape Turtle Dove & 2 & 0.2 \\
\hline Bearded Vulture & 1 & 0.1 \\
\hline African Marsh Harrier & 1 & 0.1 \\
\hline Black Harrier & 1 & 0.1 \\
\hline Lanner Falcon & 1 & 0.1 \\
\hline Pied Kingfisher & 1 & 0.1 \\
\hline Cape Eagle Owl & 1 & 0.1 \\
\hline Rock Pipit & 1 & 0.1 \\
\hline Spotted Prinia & 1 & 0.1 \\
\hline Grey-headed Sparrow & 1 & 0.1 \\
\hline Yellow Canary & 1 & 0.1 \\
\hline Mountain Chat & 1 & 0.1 \\
\hline Total & 1120 & 100 \\
\hline
\end{tabular}


Table 2

Breeding bird community of Afro-alpine Grassland in Sehlabathebe National Park in January 2001. Dominant species are given in bold case

\begin{tabular}{|c|c|c|c|c|c|c|c|}
\hline \multirow[t]{2}{*}{ Species } & \multicolumn{7}{|c|}{ Morning transects } \\
\hline & 1 & 2 & 3 & 4 & Pairs & $\mathrm{P} . / 10 \mathrm{~km}$ & Dom.(\%) \\
\hline Ayres' Cisticola & 6 & 22 & 19 & 39 & 86 & 26.9 & 11.7 \\
\hline Stonechat & 19 & 18 & 23 & 20 & 80 & 25.0 & 10.9 \\
\hline Long-tailed Widow & 4 & 13 & 14 & 12 & 43 & 13.4 & 5.8 \\
\hline Yellow-rumped Widow & 17 & 15 & 24 & 15 & 71 & 22.2 & 9.6 \\
\hline Wailing Cisticola & 17 & 11 & 4 & 10 & 42 & 13.1 & 5.7 \\
\hline Levaillant's Cisticola & 2 & 3 & 24 & 8 & 37 & 11.6 & 5.0 \\
\hline Drakensberg Siskin & 13 & 7 & 2 & 8 & 30 & 9.4 & 4.1 \\
\hline Mountain Pipit & 4 & 15 & - & 10 & 29 & 9.1 & 3.9 \\
\hline Orange-throated Longclaw & 5 & 5 & 5 & 12 & 27 & 8.4 & 3.7 \\
\hline Common Quail & - & 5 & 11 & 10 & 26 & 8.1 & 3.5 \\
\hline Black Swift & - & 4 & 5 & 16 & 25 & 7.8 & 3.4 \\
\hline Cape Bunting & 8 & 9 & 2 & 5 & 24 & 7.5 & 3.3 \\
\hline Cape Wagtail & 3 & 5 & 12 & 1 & 21 & 6.6 & 2.9 \\
\hline Sentinel Rock Thrush & 3 & 6 & 1 & 7 & 17 & 5.3 & 2.3 \\
\hline Banded Martin & 1 & 4 & 10 & 2 & 17 & 5.3 & 2.3 \\
\hline Cape Weaver & 7 & 8 & 1 & 1 & 17 & 5.3 & 2.3 \\
\hline Ground Woodpecker & 4 & 5 & 2 & 4 & 15 & 4.7 & 2.0 \\
\hline Cape Canary & 12 & 3 & - & - & 15 & 4.7 & 2.0 \\
\hline Greater Striped Swallow & 3 & 2 & 3 & 3 & 11 & 3.4 & 1.5 \\
\hline Orange-breasted Rockjumper & 2 & 1 & 2 & 6 & 11 & 3.4 & 1.5 \\
\hline Quail Finch & 1 & 4 & - & 6 & 11 & 3.4 & 1.5 \\
\hline Jackal Buzzard & 1 & 4 & 3 & 1 & 9 & 2.8 & 1.2 \\
\hline White-breasted Swallow & 4 & 1 & 2 & - & 7 & 2.2 & 1.0 \\
\hline Cape Robin & 3 & 2 & - & - & 5 & 1.6 & 0.7 \\
\hline Malachite Sunbird & 3 & 1 & - & 1 & 5 & 1.6 & 0.7 \\
\hline Red-collared Widow & 3 & 1 & - & 1 & 5 & 1.6 & 0.7 \\
\hline Grey-wing Francolin & 1 & - & - & 3 & 4 & 1.3 & 0.5 \\
\hline White-rumped Swift & - & - & - & 4 & 4 & 1.3 & 0.5 \\
\hline Yellow-breasted Pipit & 1 & 1 & 1 & 1 & 4 & 1.3 & 0.5 \\
\hline Black Duck & - & - & 2 & 1 & 3 & 0.9 & 0.4 \\
\hline Rock Pigeon & 1 & - & - & 2 & 3 & 0.9 & 0.4 \\
\hline Rock Martin & 1 & 1 & 1 & - & 3 & 0.9 & 0.4 \\
\hline Hadeda Ibis & - & 1 & 1 & - & 2 & 0.6 & 0.3 \\
\hline Yellow-billed Duck & - & - & 1 & 1 & 2 & 0.6 & 0.3 \\
\hline Rock Kestrel & - & - & 1 & 1 & 2 & 0.6 & 0.3 \\
\hline Black Crow & 1 & - & - & 1 & 2 & 0.6 & 0.3 \\
\hline Red-winged Starling & 1 & - & - & 1 & 2 & 0.6 & 0.3 \\
\hline Cape Sparrow & 1 & 1 & - & - & 2 & 0.6 & 0.3 \\
\hline Bearded Vulture & - & - & 1 & - & 1 & 0.3 & 0.1 \\
\hline African Marsh Harrier & - & - & - & 1 & 1 & 0.3 & 0.1 \\
\hline Black Harrier & 1 & - & - & - & 1 & 0.3 & 0.1 \\
\hline Lanner Falcon & - & - & - & 1 & 1 & 0.3 & 0.1 \\
\hline Cape Turtle Dove & 1 & - & - & - & 1 & 0.3 & 0.1 \\
\hline Pied Kingfisher & - & - & 1 & - & 1 & 0.3 & 0.1 \\
\hline Cape Eagle Owl & 1 & - & - & - & 1 & 0.3 & 0.1 \\
\hline Rock Pipit & 1 & - & - & - & 1 & 0.3 & 0.1 \\
\hline Spotted Prinia & 1 & - & - & - & 1 & 0.3 & 0.1 \\
\hline Pied Starling & - & 1 & - & - & 1 & 0.3 & 0.1 \\
\hline Common Waxbill & - & - & 1 & - & 1 & 0.3 & 0.1 \\
\hline Grey-headed Sparrow & 1 & - & - & - & 1 & 0.3 & 0.1 \\
\hline Yellow Canary & 1 & - & - & - & 1 & 0.3 & 0.1 \\
\hline Total & & & & 736 & & & \\
\hline
\end{tabular}


rumped Widow and Wailing Cisticola (Table 1). Together they comprised $37.9 \%$ of all breeding pairs recorded. Subdominants, represented by 15 species (Table 1), constituted another $48 \%$. Drakensberg Siskin, Mountain Pipit, Orange-breasted Rockjumper, Banded Martin and Sentinel Rock Thrush can be classified as characteristic species.
Six species (Ayres' Cisticola, Stonechat, Long-tailed Widow, Yellow-rumped Widow, Wailing Cisticola and Levaillant's Cisticola) exceeded the density of 10 pairs/100 ha, 23 species nested in a density ranging from 1 to 10 pairs $/ 10 \mathrm{~km}$. The remaining species nested in a density lower than 1 pair/10 km (Table 2).

Table 3

Breeding bird communities in farmland bordering Sehlabathebe National Park in January 2001.

Dominant species are given in bold case

\begin{tabular}{|c|c|c|c|c|}
\hline \multirow[t]{2}{*}{ Species } & \multicolumn{2}{|c|}{ Transect 1} & \multicolumn{2}{|c|}{ Transect 2} \\
\hline & Pairs & Dom. $(\%)$ & Pairs & Dom. $(\%)$ \\
\hline Cape Weaver & 2 & 1.6 & 25 & 14.0 \\
\hline Cape Sparrow & 10 & 7.9 & 13 & 7.3 \\
\hline Cape Canary & 8 & 6.3 & 13 & 7.3 \\
\hline Common Quail & 16 & 12.6 & 4 & 2.2 \\
\hline Stonechat & 7 & 5.5 & 13 & 7.3 \\
\hline Cape Bunting & 10 & 7.9 & 8 & 4.5 \\
\hline Drakensberg Siskin & 7 & 5.5 & 9 & 5.1 \\
\hline Cape Wagtail & 4 & 3.1 & 12 & 6.7 \\
\hline Mountain Pipit & 12 & 9.4 & 1 & 0.6 \\
\hline Ayres' Cisticola & 6 & 4.7 & 7 & 3.9 \\
\hline Yellow-rumped Widow & 4 & 3.1 & 8 & 4.5 \\
\hline Common Waxbill & 4 & 3.1 & 5 & 2.8 \\
\hline Long-tailed Widow & 5 & 3.9 & 3 & $\begin{array}{l}2.0 \\
1.7\end{array}$ \\
\hline Banded Martin & - & 3. & 8 & 4.5 \\
\hline Black-throated Martin & - & - & 7 & 3.9 \\
\hline Red-collared Widow & 2 & $1 . \overline{6}$ & 4 & 2.2 \\
\hline Greater Striped Swallow & - & - & 6 & 3.4 \\
\hline Wailing Cisticola & 4 & 3.1 & 1 & 0.6 \\
\hline Sentinel Rock Thrush & 3 & 2.4 & 1 & 0.6 \\
\hline Pied Starling & 4 & 3.1 & - & - \\
\hline White-breasted Swallow & - & - & 4 & 2.2 \\
\hline Malachite Sunbird & 1 & 0.8 & 3 & 1.7 \\
\hline Sentinel Rock Thrush & 3 & 2.4 & 1 & 0.6 \\
\hline Pin-tailed Whydah & 2 & 1.6 & 1 & 0.6 \\
\hline Cape Robin & 1 & 0.8 & 2 & 1.1 \\
\hline Rock Martin & - & - & 3 & 1.7 \\
\hline Black Duck & - & - & 2 & 1.1 \\
\hline Jackal Buzzard & 1 & 0.8 & 1 & 0.6 \\
\hline Horus Swift & 1 & 0.8 & 1 & 0.6 \\
\hline Rock Pigeon & - & - & 2 & 1.1 \\
\hline Quail Finch & 2 & 1.6 & - & - \\
\hline House Sparrow & 2 & 1.6 & - & - \\
\hline Grey-headed Sparrow & 2 & 1.6 & - & - \\
\hline Black Swift & - & - & 2 & 1.1 \\
\hline Red Bishop & - & - & 2 & 1.1 \\
\hline Black Crow & 1 & 0.8 & - & - \\
\hline Hadeda Ibis & 1 & 0.8 & - & - \\
\hline Hamerkop & - & - & 1 & 0.6 \\
\hline Rock Kestrel & - & - & 1 & 0.6 \\
\hline Cape Turtle Dove & 1 & 0.8 & - & - \\
\hline Ground Woodpecker & - & - & 1 & 0.6 \\
\hline Thick-billed Lark & 1 & 0.8 & - & - \\
\hline Mountain Chat & - & - & 1 & 0.6 \\
\hline Red-winged Starling & - & - & 1 & 0.6 \\
\hline Levaillant's Cisticola & - & - & 1 & 0.6 \\
\hline Total & 127 & 100.0 & 178 & 100.2 \\
\hline
\end{tabular}


Table 4

Temporal distribution of birds in Sehlabathebe National Park

Species

Dabchick

Grey Heron

Black-headed Heron

Hamerkop

Black Stork

White Stork

Sacred Ibis

Bald Ibis

Hadeda Ibis

Spur-winged Goose

Egyptian Goose

Black Duck

Yellow-billed Duck

Secretarybird

Cape Vulture

Lanner Falcon

Rock Kestrel

Yellow-billed Kite

Black Eagle

Bearded Vulture

Jackal Buzzard

Steppe Buzzard

African Marsh Harrier

Black Harrier

Gymnogene

Grey-winged Francolin

Quail

Red-knobbed Coot

Crowned Crane

Wattled Crane

Blue Crane

Ethiopian Snipe

Greenshank

Wood Sandpiper

Rock Pigeon

Cape Turtle Dove

Barn Owl

Spotted Eagle Owl

Black Swift

White-rumped Swift

Alpine Swift

Giant Kingfisher

Ground Woodpecker $\begin{array}{llllllllllll}\text { J } & \text { F } & \text { M } & \text { A } & \text { M } & \text { J } & \text { J } & \text { A } & \text { S } & \text { O } & \text { N } & \text { D }\end{array}$

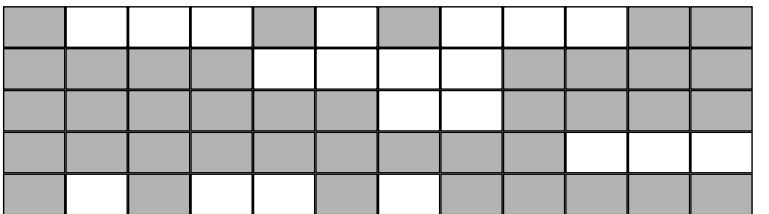


European Swallow

White-throated Swallow

Greater Striped Swallow

Rock Martin

African Sand Martin

\section{Banded Martin}

Black Crow

White-necked Raven

Rock Jumper

Cape Rock Thrush

Sentinel Rock Thrush

Mountain Chat

Stonechat

Cape Robin

Ayres' Cisticola

Wailing Cisticola

Levaillant's Cisticola

Spotted Prinia

Spotted Flycatcher

Cape Wagtail

Mountain Pipit

Yellow-breasted Pipit

Orange-thr. Longclaw

Red-winged Starling

Pied Starling

Malachite Sunbird

House Sparrow

Cape Sparrow

Cape Weaver

Red Bishop

Cape Widow

Red-collared Widow

Long-tailed Widow

Quail Finch

Pin-tailed Whydah

Drakensberg Siskin

Cape Canary

Yellow Canary

Cape Bunting

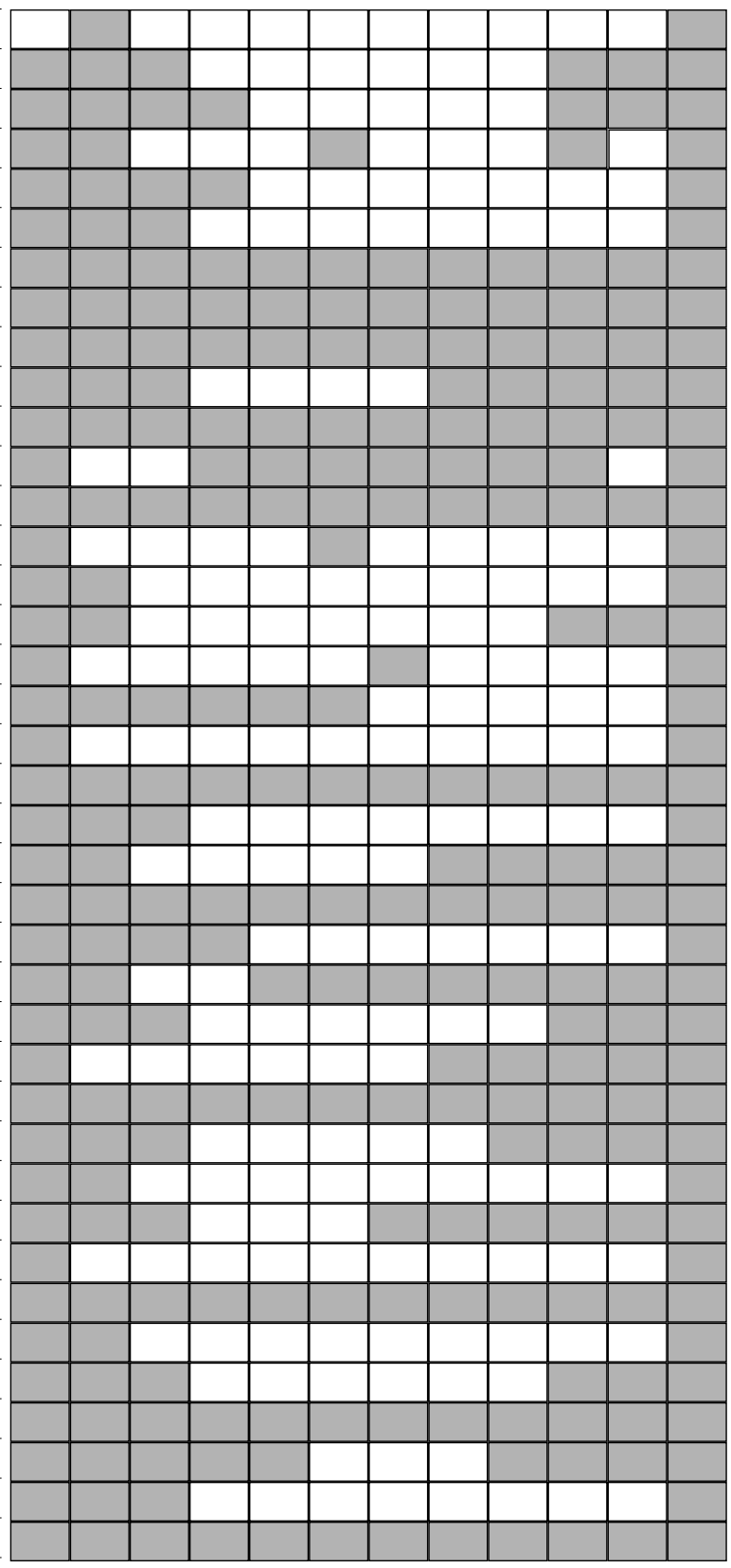


In areas bordering SNP, the dominant species were quite different. In farmland the group was composed of the Common Quail, Mountain Pipit, Cape Sparrow, Cape Bunting, Cape Canary, Stonechat and Drakensberg Siskin, while in Tsoelikane valley the dominants were the Cape Weaver, Cape Sparrow, Cape Canary, Stonechat, Cape Wagtail and Drakensberg Siskin (Table 3). Also, composition of the group of subdominant species outside the park (Table 3 ) differed markedly from that inside the park (Table 2).

\section{Annotated checklist}

Nomenclature, systematics and species numbers follow Maclean (1993).

6. Podiceps cristatus Great Crested Grebe

Vagrant. Recorded in January 1980 (J. Balcomb). This is the only Lesotho record of the species during the past 50 years.

\section{Tachybaptus ruficollis}

Dabchick Vagrant.

\section{Phalacrocorax carbo White-breasted Cormorant} Vagrant. Recorded in January 1988 (S. Fairman).

\section{Ardea cinerea}

Grey Heron

Visitor. Probably resident in neighbouring areas. On 23 December 1976, one aberrant individual with red bill and legs was observed (R. Howard).

\section{Ardea melanocephala Black-headed} Heron

Visitor. Resident in neighbouring areas.

\section{Bubulcus ibis}

Cattle Egret

Vagrant.

76. Nycticorax nycticorax Black-crowned Night Heron Vagrant.

\section{Scopus umbretta} Hamerkop
83. Ciconia ciconia White Stork

Palearctic migrant (Table 4). Most birds forage in the Tsoelikane valley below the waterfall, e.g. from 12 to 15 January 2001, a flock of 35 birds were observed there, and the whole population in the park was estimated at 40-50 pairs.

84. Ciconia nigra

Black Stork

Visitor. Resident in neighbouring areas.

91. Threskiornis aethiopicus Sacred Ibis Vagrant.

\section{Geronticus calvus} Bald Ibis Visitor. Resident in neighbouring areas.

94. Bostrychia hagedash Hadeda Ibis Visitor. Breeding in neighbouring areas.

95. Platalea alba African Spoonbill Vagrant.

\section{Alopochen aegyptiacus}

Egyptian Goose

Visitor. Probably resident in neighbouring areas.

104. Anas undulata Yellow-billed Duck Probable resident on the Tsoelikane River; uncommon.

105. Anas sparsa African Black Duck Resident on the Tsoelikane River; uncommon.

108. Anas erythrorhyncha Red-billed Teal

Vagrant. Recorded in January 1980 (J. Balcomb), 01 October 1989 (J. Tigar \& P. Osborne).

116. Plectropterus gambensis

Spur-winged Goose

Visitor. Probably resident in neighbouring areas.

118. Sagittarius serpentarius Secretarybird Visitor. Probably resident in neighbouring areas.

Bearded Vulture 
Resident at Thaba Ntšo, where one pair nests.

\section{Gyps coprotheres}

Cape Vulture

Visitor. Resident in neighbouring areas. Until late 1980's there was a breeding colony of 100-200 individuals in SNP (Barnes 1998).

126. Milvus migrans Yellow-billed Kite Vagrant. Recorded on 01 September 1985 (H. Wilson).

\section{Elanus caeruleus}

Black-shouldered Kite

Vagrant. Recorded in February 1990 (R. Guy) and November 1990 (J. Jones).

\section{Aquila verreauxii Black Eagle} Vagrant. Recorded in January 1977 (R. Howard). During the years 1986-89 a pair was recorded; probably bred at Thaba Ntšo (Osborne \& Tigar 1990).

\section{Buteo buteo Steppe Buzzard} Palearctic migrant; uncommon.

\section{Buteo rufofuscus Jackal Buzzard}

Uncommon resident.

\section{Melierax canorus}

Pale Chanting Goshawk

Vagrant. Single birds were recorded in April 1976 and December 1979 (J. Belcomb).

\section{Circus ranivorus}

African Marsh Harrier

Probable altitudinal migrant (Table 4). During the years 1975-80, only one record (28 October 1976; J. Balcomb). On 15 January 2001 an adult with two juveniles was observed 3-4 km south of the lodge (pers. obs.).

\section{Circus maurus}

Black Harrier

Probable altitudinal migrant (Table 4). Single pairs probably nested, at least during 1978/79 (J. Balcomb), 1980/81 (C. Brown), 1989/90 (R. Guy) and 2000/01 breeding seasons (pers. obs.).
Vagrant.

172. Falco biarmicus

Lanner Falcon

Rare resident.

\section{Falco tinnunculus Rock Kestrel}

Uncommon resident.

\section{Francolinus africanus}

Grey-wing Francolin

Fairly common resident in grassy areas.

200. Coturnix coturnix Common Quail Common altitudinal migrant (Tables 4 \& 5).

Table 5

Habitat selection by Common Quails (number of breeding pairs)

\begin{tabular}{lcc}
\hline Habitat & SNP & Neighbouring \\
\hline area & & \\
Grasses & 14 & 5 \\
Sedges & 5 & - \\
Grasses + sedges & 4 & - \\
Marsh & 3 & - \\
Wheat & - & 3 \\
Peas & - & 2 \\
\hline
\end{tabular}

\section{Numida meleagris}

Helmeted Guineafowl

Vagrant.

\section{Bugeranus carunculatus}

Wattled Crane

Probably 1-2 breeding pairs. A pair, 5 January 1977 (R. Howard). January, April, December 1976; March 1980 (J. Balcomb); 3-4 January 1976, December 1982 (D. Cochlan). No records since 1983. SNP is the only locality in Lesotho where the species has been recorded.

208. Anthropoides paradiseus Blue Crane Probably 1-2 breeding pairs. A pair, 28 December 1976 (R. Howard). September, 1975, October, December 1976 (J. Belcomb), 3 January 1976 (D. Cochlan). Not recorded after 1976. 
209. Balearica regulorum Crowned Crane Vagrant. Probably single pairs bred irregularly in summer 1975/76 (J. Balcomb). SNP is the only locality in Lesotho where the species has been recorded.

211. Crex crex

Corncrake

Vagrant. Recorded on 3 January 1976 (Bonde 1990).

228. Fulica cristata Red-knobbed Coot

Visitor.

\section{Neotis denhami Stanley's Bustard} Vagrant. Recorded in February and March 1979 (J. Balcomb).

264. Actitis hypoleucos Common Sandpiper Palearctic migrant (Table 4).

266. Tringa glareola Wood Sandpiper Vagrant. Two birds were recorded on 6 January 1977.

269. Tringa stagnatilis Marsh Sandpiper Vagrant. Recorded in November 1990 (S. Fairman, J. Cambridge).

270. Tringa nebularia Greenshank Palearctic migrant (Table 4).

\section{Calidris minuta} Little Stint

Palearctic migrant (Table 4). Recorded on 8 January 1977.

284. Philomachus pugnax

Ruff

Palearctic migrant (Table 4). Recorded on 29 December 1976 (D. Cochlan).

\section{Gallinago nigripennis}

Ethiopian Snipe

Probable altitudinal migrant (Table 4). Recorded in March 1977, January, February, June 1980 (J. Balcomb), November 1990.

297. Burhinus capensis Spotted Dikkop Vagrant. Recorded in 5 January 1977.

\section{Columba guinea Rock Pigeon}

Fairly common resident, nesting on sandstone cliffs.

\section{Streptopelia capicola}

Cape Turtle Dove

Rare resident in larger willow clumps.

382. Clamator jacobinus Jacobin Cuckoo Vagrant. Recorded in November 1978 (J. Belcomb).

392. Tyto alba

Barn Owl

Visitor. Resident in Sehlabathebe village (Osborne \& Tigar 1990).

400. Bubo capensis Cape Eagle Owl Probable resident, 1-2 pairs on Thaba Ntšo.

401. Bubo africanus Spotted Eagle Owl Probable resident, 1-2 pairs on Thaba Ntšo.

\section{Apus barbatus}

Black Swift

Common intra-African migrant. Nesting colonies were localised mainly around Thaba Ntšo and on sandstone cliffs around the waterfall.

415. Apus caffer White-rumped Swift Fairly common Intra-African migrant (Table 4).

416. Apus horus

Horus Swift

Uncommon intra-African migrant (Table 4), nesting along sandstone cliffs.

\section{Apus melba}

Alpine Swift

Visitor. Intra-African migrant to neighbouring areas (Table 4).

428. Ceryle rudis Pied Kingfisher Probable resident on the Tsoelikane River below the waterfall. Not recorded since 1976 (R. Howard).

429. Megaceryle maxima Giant Kingfisher Probable resident on the Tsoelikane River.

431. Alcedo cristata Malachite Kingfisher Probable resident on the Tsoelikane River.

480. Geocolaptes olivaceus

Ground Woodpecker 
Common resident. Breeds in the banks of rivers and streams and in erosion gulleys.

500. Mirafra curvirostris Long-billed Lark Probable resident; rare.

507. Calandrella cinerea Red-capped Lark Probably rare nomadic.

512. Galerida magnirostris

Thick-billed Lark

Rare resident.

518. Hirundo rustica European Swallow Palearctic migrant (Table 4).

520. Hirundo albigularis

White-throated Swallow Intra-African migrant (Table 4); uncommon.

\section{Hirundo cucullata}

Greater Striped Swallow Intra-African migrant (Table 4). Present along sandstone cliffs and on Thaba Ntšo.

\section{Hirundo fuligula}

Rock Martin

Fairly common altitudinal migrant (Table 4); nesting on sandstone cliffs.

\section{Delichon urbica House Martin} Palearctic migrant (Table 4). Recorded in February 1990 (R. Guy).

\section{Riparia paludicola}

Brown-throated Martin

Fairly common resident, nesting mainly along Tsoelikane River.

\section{Riparia cincta}

Banded Martin

Fairly common Intra-African migrant, nesting mainly along Tsoelikane River.

\section{Corvus capensis}

Black Crow

Resident; 2-5 pairs on larger sandstone cliffs and at Thaba Ntšo.

548. Corvus albus Pied Crow

Resident; 1-2 pairs at Thaba Ntšo.

550. Corvus albicollis

White-necked Raven
Probably resident. One probable breeding pair at Thaba Ntšo.

551. Parus afer

Southern Grey Tit Vagrant. Recorded on December 1988 (S. Fairman).

\section{Monticola rupestris}

Cape Rock Thrush Rare resident; nesting along sandstone cliffs.

\section{Monticola explorator}

Sentinel Rock Thrush Common resident in short grasses with rocky outcrops or along rocky cliffs.

586. Oenanthe monticola Mountain Chat Probable rare resident.

589. Cercomela familiaris Familiar Chat Probable rare resident.

\section{Cercomela sinuata}

Sickle-winged Chat Visitor. Breeding in neighbouring areas.

596. Saxicola torquata Stonechat Most birds are altitudinal migrants, some are resident. Very common in grassland, marshes and along streams and valleys during the breeding season. Breeds mainly in December/January, e.g. on 12-15 January 2001, most pairs were seen with 2-3 juveniles.

601. Cossypha caffra

Cape Robin Fairly common resident in willow clumps.

\section{Chaetops aurantius}

Orange-breasted Rockjumper Fairly common resident; nesting along sandstone formations.

667. Cisticola ayresii Ayres' Cisticola Very common altitudinal migrant; breeding mainly in short grass.

670. Cisticola lais Wailing Cisticola Very common resident, mainly in shorter grasses on sloping hillsides. 
677. Cisticola tinniens

Levaillant's Cisticola

Very common resident in marshes and longer grasses, especially along water courses.

686. Prinia hypoxantha Spotted Prinia Rare resident on lower slopes of Thaba Ntšo.

654. Muscicapa striata Spotted Flycatcher Palearctic migrant. Recorded on December 1977 and January 1980 (J. Belcomb).

698. Sigelus silens

Fiscal Flycatcher

Vagrant.

\section{Motacilla capensis Cape Wagtail} Common resident along water courses, at rock pools and dams.

701. Anthus hoeschi Mountain Pipit Very common intra-African migrant, nesting mainly in short grass.

721. Anthus crenatus Rock Pipit Probable resident on lower rocky slopes of Thaba Ntšo.

\section{Hemimacronyx chloris}

Yellow-breasted Pipit

Uncommon altitudinal migrant (Table 4 ); breeding in short grass.

\section{Macronyx capensis}

Orange-throated Longclaw Common resident in marshes and in grass, especially along streams and river valleys.

\section{Lanius collaris}

Fiscal Shrike

Visitor. Resident in neighbouring areas.

759. Spreo bicolor

Pied Starling

Uncommon resident on lower slopes of Thaba Ntšo.

\section{Onychognathus morio}

Red-winged Starling Altitudinal migrant (Table 4); nesting along sandstone cliffs; uncommon. December-April.
775. Nectarinia famosa Malachite Sunbird Fairly common altitudinal migrant (Table 4), usually close to sandstone formations and Kniphofia and Aloe communities.

801. Passer domesticus House Sparrow Visitor, Resident at Sehlabathebe village.

803. Passer melanurus Cape Sparrow Uncommon resident. Nesting recorded in old Cape Weaver nests in willows.

804. Passer diffusus Grey-headed Sparrow Rare resident recorded in willow clumps.

813. Ploceus capensis Cape Weaver Common altitudinal migrant (Table 4). Nests were located exclusively in willows, both in SNP and in the neighbouring area. Outside the park, on the banks of the Tsoelikane River, most nests were located 1-2 $\mathrm{m}$ above the water level. The number of nests per group ranged from 3 to 20 .

824. Euplectes orix $\quad$ Red Bishop Probable altitudinal migrant (Table 4); rare. Breeding was recorded in old Cape Weaver nests in willows on banks of the Tsoelikane River outside SNP. This nest site is unusual for this species.

826. Euplectes afer Golden Bishop Probably altitudinal migrant (Table 4); rare.

827. Euplectes capensis Yellow-rumped Widow Very common altitudinal migrant (Table 4); breeding in marshes and in grass along water-courses and at dams.

\section{Euplectes ardens}

Red-collared Widow

Fairly common altitudinal migrant (Table 4); breeding usually in marshes.

832. Euplectes progne Long-tailed Widow 
Very common resident in marshes and in grass along water-courses and at dams.

\section{Estrilda astrild Common Waxbill}

Fairly common resident, mainly along the Tsoelikane River.

852. Ortygospiza atricollis Quail Finch Fairly common altitudinal migrant (Table 4); breeding in grass, usually in close proximity of pools and dams.

860. Vidua macroura Pin-tailed Whydah Uncommon altitudinal migrant (Table 4).

\section{Serinus canicollis Cape Canary} Common altitudinal migrant (Table 4); breeding in grassy areas.

\section{Pseudochloroptila symonsi}

Drakensberg Siskin

Very common resident; breeding in grassy areas and marshes. Feeds mainly on seeds of Helichrysum spp. and Polygonum spp. A nest with one egg (20.5 x $16.5 \mathrm{~mm})$ was found on 13 January 2001, located in short grass mixed with numerous Helichrysum flowers.

\section{Serinus flaviventris Yellow Canary} Probable altitudinal migrant (Table 4); rare.

\section{Emberiza capensis Cape Bunting}

Very common resident, usually along sandstone formations.

\section{Emberiza tahapisi Rock Bunting}

Vagrant. Recorded on 1 September 1985 (H. Wilson), 13 March 1987 (C. Brown).

The following species need confirmation before their inclusion into the list: Little Swift Apus affinis, Whinchat Saxicola rubetra White-winged Widow Euplectes albonotatus and Bully Canary Serinus sulphuratus. All these species were listed by Passineau (1977), but without corroborating evidence. They are regarded as very rare in Lesotho highlands (Osborne \& Tigar 1990, Bonde 1993) and can be confused with congeneric species. The records may be considered, therefore, to derive from misidentification.

\section{Conclusions}

To date 117 bird species have been recorded in the SNP, 29 of them are vagrants, 18 are visitors, and 70 are breeding or probable breeding residents.

Dominant species are represented by Stonechat, Ayres' Cisticola, Yellow-rumped Widow and Wailing Cisticola. There are 15 subdominant species. Characteristic species include Drakensberg Siskin, Mountain Pipit, Orange-breasted Rockjumper, Banded Martin and Sentinel Rock Thrush.

It is important to note the absence or very low densities of the following species in SNP, which are widespread and common in the Maloti/Drakensberg grasslands (including areas neighbouring to SNP): Laughing Dove Streptopelia senegalensis, Sicklewinged Chat, Mountain Chat, Thick-billed Lark, Red-winged Starling, Alpine Swift, Cape Sparrow, Grey-headed Sparrow, Red Bishop and Golden Bishop (cf. Osborne \& Tigar 1990, Little \& Bainbridge 1992, Bonde 1993). The lack of trees and shrubs for nesting, the lack of cultivated fields as feeding places and competition with related species both for food and nesting sites, may partly play a role in this regard.

\section{Acknowledgements}

This study was sponsored by the RCC Committee of the National University of Lesotho. Dr D. N. Johnson, Prof. O. B. Kok and Mr R. J. Nuttall are thanked for reviewing the paper. My thanks are also due to Prof D. Ambrose for indicating some unpublished reports. I also thank the Administration of the SNP for providing me accommodation during this study. 


\section{References}

BALcomb, J. 1980. Birds sighted in Sehlabathebe National Park, 1975-1980. Unpublished internal report.

BARNES, K. N. 1988. Important bird areas of Lesotho. Pp. 281-294. In: BARNES, K. N. (ed.). The Important Bird Areas of Southern Africa. Johannesburg: BirdLife South Africa.

BibBy, C.L., N.D. Burgess \& D.A.Hill. 1992. Bird Census Techniques. London: Academic Press.

Bonde, K. 1993. Birds of Lesotho. A guide to distribution past and present. Pietermaritzburg: University of Natal Press.

Brown, C.J. \& P.R. BARnes. 1984. Birds of the Natal Alpine Belt. Lammergeyer 33: 1-13.

HowarD, R. 1977. Lesotho bird list. Unpublished internal report.

Howard, R. \& M. Howard. 1977. Report on ornithological work, [Sehlabathebe National Park], January 2 to 9, 1977. Unpublished report.

Koskimies, P. \& R.A. VÄISÄNEN. 1991. Monitoring Bird Populations. Helsinki: Zoological Museum, Finnish Museum of Natural History.
KopIJ, G. 2000. Birds of Maseru. NUL Journal of Research 8: 104-151.

KopIJ, G. 2001a. Birds of Roma Valley, Lesotho. Roma: National University of Lesotho.

KopIJ, G. 2001b. Sehlabathebe National Park. Roma: National University of Lesotho.

LitTLE, R.M. \& W.R. BAinBRidge. 1992. Birds of the Natal Drakensberg Parks. Pietermaritzburg: Wildlife Society.

LYNCH, C.D. \& J.P. Watson. 1990. The mammals of Sehlabathebe National Park, Lesotho. Navorsinge van die Nasionale Museum, Bloemfontein 6: 523-554.

Osborne, P.E. \& B.J. Tigar. 1990. The status and distribution of birds in Lesotho. Unpublished report.

Passineau, L. 1977. Birds of Sehlabathebe National Park. Maseru: Lesotho National Parks.

Pomela, E.M., C. Mokuku, S. 'Mabataung, R. Phororo, M. Mokoae, M. Majoro, D. May, D. Ambrose \& S. TAluKdar. 2000. Biological Diversity in Lesotho: a Country Study. Maseru: Lesotho Government, National Environmental Secretariat. 